\title{
Customized versus conventional video counseling for peritoneal dialysis decision-making in patients with stage 5 chronic kidney disease under a PD-first policy: a randomized controlled study
}

\author{
Watanyu Parapiboon ${ }^{(i)}$, Wannapat Pitsawong ${ }^{(\mathbb{D})}$, Laddaporn Wongluechai ${ }^{(\mathbb{D}}$, Kanin Thammavaranucupt $^{(\mathbb{D})}$, \\ Lalana Raegasint
}

Department of Medicine, Maharat Nakhon Ratchasima Hospital, Nakhon Ratchasima, Thailand

Background: Indecision regarding the start of peritoneal dialysis (PD) is a challenging problem in chronic kidney disease (CKD) stage 5 patients who receive conventional video counseling. This study aimed to evaluate the effect of video counseling customized to the local context versus conventional video counseling on PD decision-making in CKD stage 5 patients under PD-first policy.

Methods: We enrolled 120 patients with stage 5 CKD in Thailand who initiate PD between May 2016 to January 2017 in a randomized, open-label, controlled study. Patients were randomized to either a customized or conventional video counseling group. The primary outcome was PD acceptance rate with complete PD catheter insertion on schedule. The secondary outcomes were change in patient knowledge and confidence in PD and reasons for indecision PD.

Results: We analyzed 120 patients (customized, $n=60$ vs. conventional, $n=60$ ). The two groups were similar for age (55 vs. 56 years), blood urea nitrogen ( 89 vs. $86 \mathrm{mg} / \mathrm{dL}$ ), creatinine (10.37 vs. $11.29 \mathrm{mg} / \mathrm{dL}$ ), and eGFR (4.7 vs. $5.6 \mathrm{~mL} / \mathrm{min} / 1.73 \mathrm{~m}^{2}$ ). The PD acceptance rate along with PD catheter insertion on schedule in the customized video counseling group was not significantly different from that in the conventional video counseling group $(66.6 \%$ vs. $63.3 \%$, relative risk: $0.97,95 \%$ confidence interval: 0.73 to 1.29; $P=0.86$ ). Patient knowledge of and confidence in PD increased after counseling, but the difference was not significant.

Conclusion: Among stage 5 CKD patients, counseling content customized to a local context did not differ in a rate of acceptance for beginning PD with PD catheter insertion on schedule compared with conventional video counseling.

Keywords: Counselling, Peritoneal dialysis, Peritoneal dialysis-first policy, Randomized controlled trial

Received March 8, 2020; Revised July 6, 2020;

Accepted July 20, 2020

Editor: Chan-Duck Kim, Kyungpook National University, Daegu, Republic of Korea

Correspondence: Watanyu Parapiboon

Department of Medicine, Maharat Nakhon Ratchasima Hospital, 49 Cheng Pek Road, Nakhon Ratchasima 30000, Thailand. E-mail: watanyupara@gmail.com

Copyright (@ 2020 by The Korean Society of Nephrology

(a) This is an open-access article distributed under the terms of the Creative Commons Attribution Non-Commercial License (http://creativecommons. org/licenses/by-nc-nd/4.0/), which permits unrestricted non-commercial use, distribution, and reproduction in any medium, provided the original work is properly cited.

\section{Introduction}

Education and dialysis decision aids are necessary for patients with chronic kidney disease (CKD), especially when they reach stage 4 [1]. Early and effective dialysis counseling can help smooth the transition from CKD to a planned start of renal replacement therapy, improve symptom control, and even improve mortality [2-4]. The general concept of counseling for dialysis modality should be based on a shared decision-making process among physicians, patients, and caregivers $[5,6]$ and should be timely to prevent an unplanned start to dialysis 
that leads to poor patient outcomes [7].

Interestingly, Thailand's universal coverage program has a unique concept for selecting the dialysis modality called a PD-first policy, which is used in approximately $75 \%$ of the total population $[8,9]$. Continuous ambulatory peritoneal dialysis (CAPD) is the first free modality of choice in stage 5 CKD patients who are eligible for peritoneal dialysis (PD). However, effective education and counseling about starting PD in a timely manner that actually leads to an as-planned start to PD is still a major challenge.

Effective educational content for PD modality should fit patient lifestyle, home environment, and sociocultural status. Moreover, effective education materials may help patients and caregivers overcome any psychosocial barriers to starting PD, such as anxiety about making a major life decision and lack of confidence about performing PD at home. Therefore, education and counseling aids should be customized and comprehensive for the diversity of patient contexts and concerns. Interestingly, evidence regarding the method and content of education and counseling aids (which help patients accept PD in a timely manner and begin PD without temporary hemodialysis) is lacking, especially in the PD-first setting. We hypothesized that customized counseling that has been tailored to the context of the patients in our region would help increase PD acceptance. The main objective of this study was to compare a customized video counseling session (tailored to patient context) with a conventional video counseling session (for general use) to determine the acceptance rate of dialysis decision-making in stage 5 CKD patients under a PD-first policy.

\section{Methods}

\section{Study design}

We conducted a randomized, open-label, controlled clinical trial between May 2016 and January 2017 at a tertiary care referral hospital in northeastern Thailand. All procedures performed in studies involving human participants were in accordance with the ethical standards of the Maharat Nakhon Ratchasima Ethics Committee of Human Research, Maharat Nakhon Ratchasima Hospital, Thailand. Approval number: 031/2016. Informed written consent was obtained from all the participants or their family members before the study began. This study was registered under the Thai Clinical Trial Registry number TCTR20190606003 which followed the World Health Organization International Clinical Trials Registry Platform (WHO-ICTRP) dataset.

\section{Patients and randomization}

Patients from 18 to 75 years of age who had stage 5 CKD and who were expected to begin PD within four to six weeks in a universal coverage program with a PD-first policy were enrolled from both outpatient and inpatient settings by a nephrologist. The participants were randomly assigned by a PD nurse into blocks of four; patients were given a sealed opaque envelope and had an equal chance of receiving either conventional or customized video counseling about their final PD decisionmaking. Patients with clinical contraindications to PD (a lower midline abdominal scar from a previous surgery, severe psychological problem, blindness in both eyes, abdominal or inguinal hernia, body mass index $>35 \mathrm{~kg} /$ $\mathrm{m}^{2}$ ) or obvious social barriers to PD (e.g., homelessness, living alone with a disability) were changed to hemodialysis and excluded. Patients with advanced malignancy or a life expectancy less than three months were offered palliative care and excluded. Finally, any participants who had previously been exposed to video counseling, who were willing to choose hemodialysis by self-payment, and who were scheduled to undergo preemptive kidney transplant were also excluded.

Video counseling and educational program for final PD decision

Stage 5 CKD patients were followed up in the CKD clinic by a nephrologist or were referred from a network hospital by an internist or general practitioner. General counseling on living with $\mathrm{CKD}$, slowing disease progression, managing diet, managing medications, and planning for PD or kidney transplant was provided during routine CKD clinic visits beginning in stage 4 . When the estimated glomerular filtration rate (eGFR) decreased and PD was expected to begin within four to six weeks or when the patient was urgently referred from a network hospital due to CKD complications, the patient and a caregiver were sent to an educational counseling pro- 
gram for final PD decision-making. This four-step PD counseling aid protocol designed to help patients make the final decision to progress to PD was run by a PD nurse for approximately 1.5 hours. The first step involved developing a relationship with patients and family members; the second step was setting a goal for the planned start of PD and offering information on how to avoid CKD complications. The third step included the use of customized or conventional video counseling for education, and the last step involved answering any questions about or addressing obstacles patients faced due to PD. Ten questions on patient knowledge and confidence with $\mathrm{PD}$ were administered during the pretest and posttest. Consent from patients and family members for PD treatment was given after the counseling process was concluded. If the patients decided to proceed with $\mathrm{PD}$, a nephrologist placed a coiled double-cuff PD catheter using a percutaneous bedside technique within two weeks, followed by a break-in period of two weeks, and training was provided to patients and caregivers in the CAPD program for one week. Patients with severe CKD complications underwent temporary hemodialysis before the PD catheter was inserted depending on the nephrologist's judgment. After completing this process, patients were regularly followed up in the CAPD clinic. If the patients could not make a final decision about accepting PD, the PD nurse conducted an interview to determine the reasons for postponing $\mathrm{PD}$, and the patients were sent back to the CKD clinic. The patients then began counseling and joined the educational program during their next visit. During this period, the PD nurse phoned the patients to identify any CKD complications.

\section{Customized vs. conventional video counseling}

We used a video as part of the third step of the educational and counseling program. A conventional 40-minute counseling video was developed at the beginning of the PD-first policy adoption in the central part of Thailand and is currently used in daily practice. This video provides basic knowledge about CKD, choice of dialysis modality, how to run a home CAPD, and patient experience. All the content in the video was provided in the Thai language and was spoken with a formal central Thai accent. Patients who participated in the video were middle-class employed individuals from urban areas with a college degree and a positive home environment who had been chosen to convey the idea that PD was not a second-class modality of dialysis. As previously mentioned, many factors can influence decision-making, including culture, personal beliefs, home environment, and lifestyle, and the social backgrounds of patients differed based on the region of Thailand in which they lived. A 32-minute customized video that fit the region's lifestyle was created to address these specific issues. The video content was presented in the Thai language with a northeastern Thai accent and large Thai subtitles. A wide range of $\mathrm{PD}$ patient experiences that represent diverse incomes and educational backgrounds appear in the video. The participants ranged from unemployed lower-class patients with primary school education and a limited home environment to middle-class patients with an ideal home environment.

Moreover, common misconceptions and fears related to PD-related complications from the experiences of previous patients in our center who did not choose PD were clarified in this video, especially concerning PD catheter insertion and PD-related peritonitis. Finally, the consequences of indecision concerning dialysis initiation were described, including CKD complications and patient experiences of temporary hemodialysis catheter insertion. A summary of the difference between the conventional and customized multimedia videos is shown in Table 1.

\section{Outcomes}

The primary outcome was acceptance rate along with PD catheter insertion on schedule. The secondary outcomes were changes in patient knowledge and confidence in PD scores by the end of the counseling program. Pretest and posttest scores were assessed using 10 trueor-false questions. Five experts were asked to validate the questions by scoring each (with -1 : clearly not suitable, 0 : unclear, and +1 : clearly suitable) and calculating the index of item objective congruence (IOC). All 10 questions were deemed valid with an IOC score $>0.75$ [10]. The 10 true-or-false questions are shown in Supplementary Table 1 (available online). The rate of unplanned start to PD, which represented the need for temporary hemodialysis before beginning PD, and any reasons for postponing PD in each video group were also reported. 
Table 1. Content of the customized and conventional counseling videos

\begin{tabular}{lcc}
\hline \multicolumn{1}{c}{ Details of counselling } & Customized multimedia & Conventional multimedia \\
\hline Duration (min) & 32 & 40 \\
Language & Northeastern regional Thai dialects & Formal Thai language \\
& with Thai subtitles & Yes \\
Introduction to kidney disease & Yes & Yes \\
Choice of treatment for stage 5 CKD & Yes & No \\
Consequences of delaying dialysis (e.g., temporary & & Yes \\
hemodialysis, hospitalization due to volume overload) & Yes & Yes \\
Introduction to PD & Yes & Government officer, salary earner \\
How to change PD fluids & Unemployed, farmers, salary earners & Middle income \\
People who share the patient's experience & Lower to middle income & No \\
Occupation & Yes & No \\
Household income & Yes \\
Patient experience before and after PD & & Yes \\
Caregiver experience & Yes \\
Fears and barriers to PD & Yes \\
PD catheter insertion & Yes \\
PD-related peritonitis & Nell suited for a limited home environment \\
Logistics of PD fluids & No \\
Home environment & No & No \\
\hline
\end{tabular}

CKD, chronic kidney disease; PD, peritoneal dialysis.

\section{Statistical analysis}

These studies required 104 participants to detect differences between the two groups at $80 \%$ power and an alpha of 0.05 [11]. The acceptance rate along with the rate of PD catheter insertion on schedule in patients who watched the conventional video was $55 \%$. The increase to an $80 \%$ acceptance rate with on-time PD catheter insertion after the patients watched the customized video was clinically significant. Nominal variables were analyzed with Chisquare test. Continuous variables were analyzed using Student's $t$ test for analysis. Univariate and multivariate analyses by stepwise logistic regression were performed to identify potential factors for not accepting PD. The descriptive statistics were mean with standard deviation, median with interquartile range, and number with percentage. Intention-to-treat analyses were used. All the reported $P$ values were from two-sided tests, and $P<0.05$ was statistically significant. Analyses were performed using Stata Statistical Software: Release 12 (Statistical Software; StataCorp LP, College Station, TX, USA).

\section{Results}

One hundred forty-four patients who had stage 5 CKD under the PD-first policy were canvassed during the study period. We identified 128 eligible participants, and 120 of them (93.8\%) were enrolled in this study. The reasons for exclusion and the CONSORT Statement are shown in Fig. 1 (two patients were candidates for preemptive, living-related kidney transplantation; two patients were undergoing hemodialysis by self-payment; and four patients had obvious social barriers to PD). Finally, 120 participants were randomized and assigned to either a customized video counseling group or a conventional video counseling group at the beginning of a four-step protocol for an education and counseling program for final PD decisionmaking. There were no exclusions after randomization and no dropout of participants. Baseline characteristics showed no significant difference between the two groups (Table 2). Most of the participants were of low socioeconomic status ( $79 \%$ had a primary school education; $53 \%$ were unemployed and had an average household income of USD \$156 per month). More than half of the patients (62\%) participated in follow-up with a nephrologist after less than four weeks, and almost all (91\%) had symptoms 


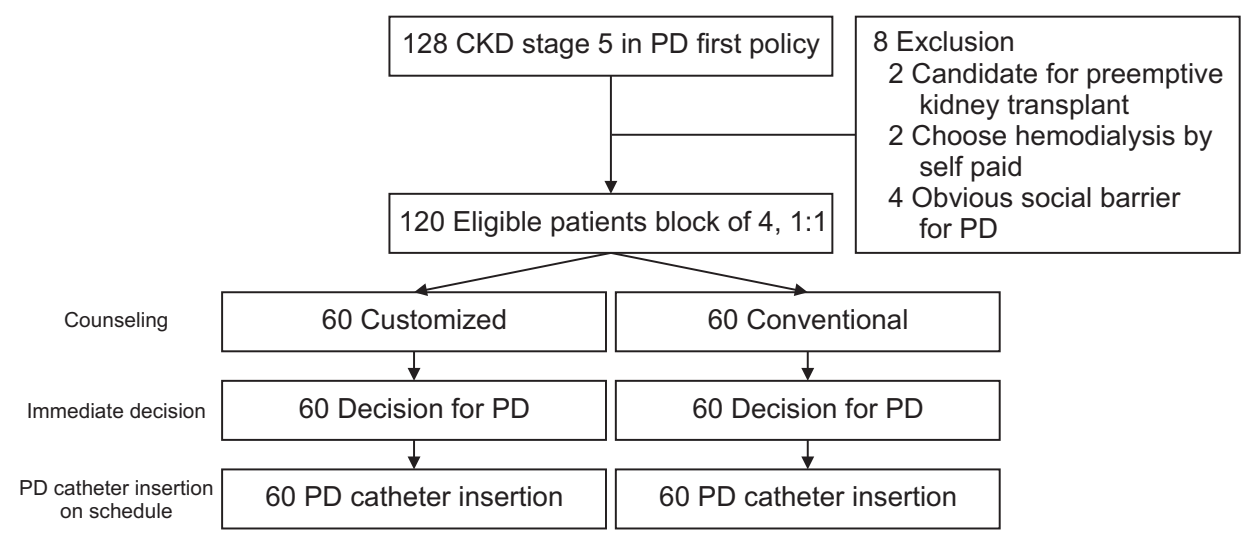

Figure 1. Study flow as a CONSORT diagram.

CKD, chronic kidney disease; PD, peritoneal dialysis.

Table 2. Baseline characteristics

\begin{tabular}{lccc}
\hline \multicolumn{1}{c}{ Characteristic } & Customized video $(\mathrm{n}=60)$ & Conventional video $(\mathrm{n}=60)$ & $P$ value \\
\hline Age (yr) & $56.2 \pm 13.4$ & $55.7 \pm 12.3$ & 0.810 \\
Male & $22(36.7)$ & $32(53.3)$ & 0.060 \\
Having a caregiver & $56(93.3)$ & $58(96.7)$ & 0.400 \\
Primary school education & $46(76.7)$ & $49(81.7)$ & 0.820 \\
Occupation & & & \\
Unemployed & $34(56.7)$ & $30(50.0)$ & 0.460 \\
Farmer & $6(10)$ & $8(13.3)$ & 0.560 \\
Employee & $8(13.3)$ & $6(10.0)$ & 0.560 \\
Household income per month (USD, \$) & $156(33,312)$ & $156(50,312)$ & 0.970 \\
DM & $33(55.0)$ & $35(58.3)$ & 0.715 \\
Symptoms of CKD & $56(93.3)$ & $54(90.0)$ & 0.513 \\
Nephrologist follow-up in fewer than three months & $44(73.3)$ & $37(61.7)$ & 0.170 \\
CKD unawareness & $37(61.7)$ & $23(38.3)$ & 0.010 \\
OPD setting & $18(30.0)$ & $22(36.7)$ & 0.440 \\
\hline
\end{tabular}

Data are presented as mean \pm standard deviation, number (\%), or median (interquartile range).

CKD, chronic kidney disease; DM, diabetes mellitus; OPD, outpatient clinic.

of CKD (uremia, history of volume overload, fatigue due to anemic symptoms) during enrollment. Laboratory data showed no significant difference between the two groups (Table 3). Blood urea nitrogen was 86 to $89 \mathrm{mg} /$ dL. Creatinine was 10 to $11 \mathrm{mg} / \mathrm{dL}$, and the eGFR was 4 to $5 \mathrm{~mL} / \mathrm{min} / 1.73 \mathrm{~m}^{2}$. Forty-three out of 60 participants accepted PD immediately after customized video counseling, and 42 out of 60 patients did so following conventional video counseling. However, the acceptance rate with PD catheter insertion on schedule was $66.7 \%$ (40 out of 60 ) in the customized video counseling group and $63.3 \%$ (38 out of 60 ) in the conventional video counseling group (Fig. 2). There were seven participants (three in the customized video counseling group and four in the conventional video counseling group) who accepted PD immediately after counseling but then did not present for
Table 3. Laboratory values before randomization

\begin{tabular}{lccc}
\hline & $\begin{array}{c}\text { Customized } \\
\text { video }(\mathrm{n}=60)\end{array}$ & $\begin{array}{c}\text { Conventional } \\
\text { video }(\mathrm{n}=60)\end{array}$ & $P$ value \\
\hline $\mathrm{Hb}(\mathrm{g} / \mathrm{dL})$ & $7.8 \pm 1.3$ & $7.7 \pm 1.6$ & 0.590 \\
$\mathrm{BUN}(\mathrm{mg} / \mathrm{dL})$ & $89.8 \pm 30.8$ & $86.8 \pm 29.5$ & 0.590 \\
Creatinine $(\mathrm{mg} / \mathrm{dL})$ & $11.29 \pm 4.9$ & $10.37 \pm 5.39$ & 0.320 \\
eGFR $\left(\mathrm{mL} / \mathrm{min} / 1.73 \mathrm{~m}^{2}\right)$ & $4.7 \pm 2.7$ & $5.6 \pm 2.8$ & 0.100 \\
$\mathrm{Na}^{+}(\mathrm{mEq} / \mathrm{L})$ & $134.9 \pm 6.3$ & $135.1 \pm 5.8$ & 0.790 \\
$\mathrm{~K}^{+}(\mathrm{mEq} / \mathrm{L})$ & $4.99 \pm 5.04$ & $4.48 \pm 0.74$ & 0.430 \\
$\mathrm{HCO}_{3}{ }^{-}(\mathrm{mEq} / \mathrm{L})$ & $19.2 \pm 4.8$ & $19.6 \pm 5.4$ & 0.630 \\
$\mathrm{Ca}^{2+}(\mathrm{mg} / \mathrm{dL})$ & $8.0 \pm 1.1$ & $7.7 \pm 1.1$ & 0.250 \\
$\mathrm{PO}_{4}{ }^{3-}(\mathrm{mg} / \mathrm{dL})$ & $6.3 \pm 2.2$ & $6.0 \pm 2.1$ & 0.410 \\
$\mathrm{Albumin}(\mathrm{g} / \mathrm{dL})^{3}$ & $3.21 \pm 1.34$ & $2.97 \pm 0.69$ & 0.070 \\
\hline
\end{tabular}

Data are presented as mean \pm standard deviation.

BUN, blood urea nitrogen; $\mathrm{Ca}^{2+}$, calcium; $\mathrm{Hb}$, hemoglobin; $\mathrm{HCO}_{3}{ }^{-}$, bicarbonate; $\mathrm{K}^{+}$, potassium; $\mathrm{PO}_{4}{ }^{3-}$, phosphate. 
PD catheter insertion on time. There was no significant difference in acceptance rate with PD catheter insertion on schedule between the customized and conventional groups (relative risk, 0.97 ; 95\% confidence interval, 0.73 to $1.29 ; P=0.860$ ). Due to indecision regarding $\mathrm{PD}$, only $10 \%$ of the customized video counseling group patients feared infection within their home environment, whereas a much larger proportion (38\%) of the conventional video counseling group feared infection. All the reasons for postponing PD are shown in Table 4.

The pretest patient knowledge of and confidence in score was assessed using a questionnaire with 10 truefalse questions and was not significantly different between the two groups ( $7.1 \pm 1.3$ vs. $6.8 \pm 1.3 ; P=0.290$ in the customized and conventional groups, respectively). Patient knowledge and confidence scores increased after video counseling and participation in an educational program in both the customized and conventional groups $(P=0.005$ and $P=0.001)$, but there was no significant difference between the two groups, as shown in Fig. 3 (7.8 \pm 1.3 vs. $7.5 \pm$

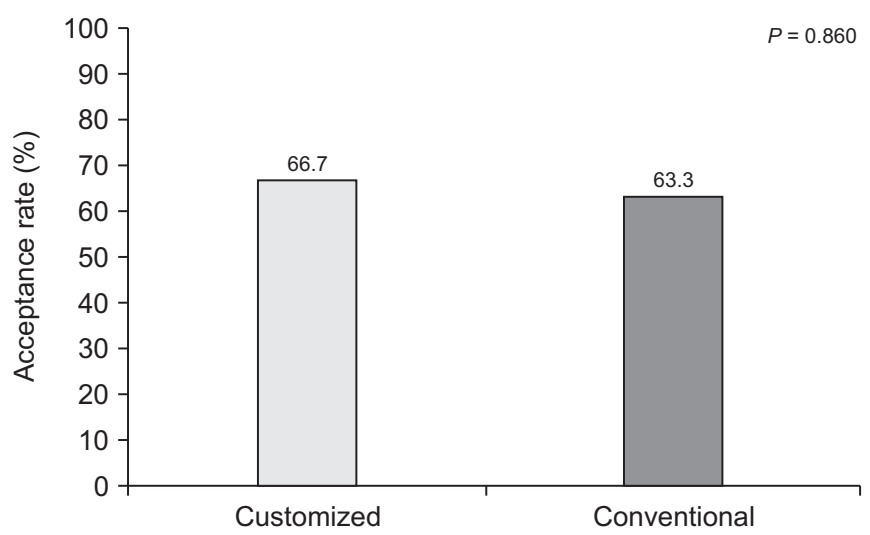

Figure 2. The acceptance rate with peritoneal dialysis (PD) catheter insertion on schedule.
1.6, respectively; $P=0.370$ ). In addition, there was no significant difference between the two groups in incidence of unplanned start to PD (30\% vs. $40 \%$ in the customized and conventional groups, respectively; $P=0.350$ ).

\section{Discussion}

In this study, customized video counseling that was tailor-made for the local context was not significantly different from conventional video counseling in terms of acceptance rate with PD catheter insertion on schedule in patients with stage 5 CKD under the PD-first policy. Moreover, patient knowledge of and confidence in PD after both customized and conventional video counseling increased, but there was no significant difference between these two groups. However, clear differences between the groups were found with respect to reasons for

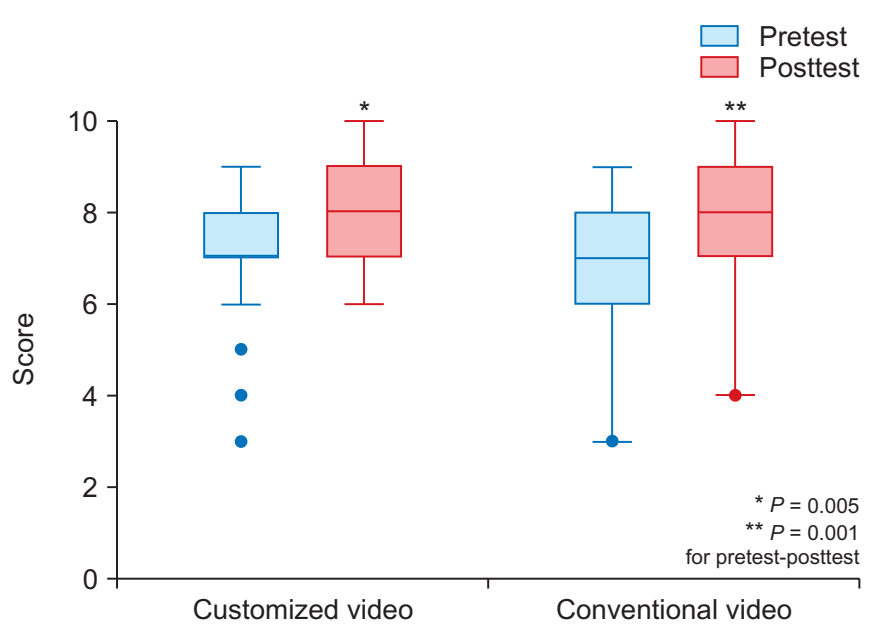

Figure 3. Pretest and posttest scores of patient knowledge of and confidence in peritoneal dialysis (PD) between the customized video and conventional video groups.

Table 4. Reasons for postponing PD in each video counseling group based on patient self-report (one patient may have multiple reasons)

\begin{tabular}{lcc}
\hline \multicolumn{1}{c}{ Reason for postponing } & $\begin{array}{c}\text { Customized video (28 reasons } \\
\text { from 17 undecided patients) }\end{array}$ & $\begin{array}{c}\text { Conventional video (34 reasons } \\
\text { from 16 undecided patients) }\end{array}$ \\
\hline I feel unhealthy but don't want to change the status quo & 8 & 5 \\
I feel healthy, so why do I need dialysis? & 2 & 1 \\
I don't want to be a burden on family members & 13 & 10 \\
I think my home is not a perfect environment for changing PD & 3 & 13 \\
fluids, so I will suffer from PD-related infectious complications & & 5 \\
I fear the PD catheter insertion procedure & 1 & 0 \\
I will die soon if I start PD & 1 & 5
\end{tabular}

$\mathrm{PD}$, peritoneal dialysis. 
postponing PD.

Using customized video counseling, we attempted to overcome the challenge of indecision regarding PD in patients with stage $5 \mathrm{CKD}$ at a suitable time point before life-threatening CKD complications occurred. The background hypothesis was that the diversity of patients among regions of the PD center, in terms of household income, home environment, lifestyle, and illness beliefs, affected their decision-making regarding PD dialysis. Our study found that the reasons for indecision in starting PD differed between the two video counseling groups. There were fewer barriers to starting PD in terms of concern for infection due to a limited home environment and fear of PD catheter insertion in the customized video counseling group than in the conventional video counseling group ( 3 vs. 13 and 1 vs. 5 for concern for infection and fear of PD catheter insertion, respectively). These findings may explain why our customized video counseling produced successful PD patients not only with average socioeconomic status and an ideal home environment, but also with low household incomes and a limited home environment. Moreover, the customized video counseling addresses common concerns about PD, especially the PD catheter insertion procedure, which helps to diminish fears among patients who postpone the decision to accept PD.

However, the acceptance rate with on-schedule PD catheter insertion among those who received the customized video counseling was not significantly different from that among those who viewed the conventional video counseling. This finding may have arisen for the following reasons. First, a one-time counseling video may not be sufficient to change a patient's attitude. Repeated education sessions using each counseling video likely influences dialysis decision-making. Second, most of the participants $(67.5 \%)$ had late presentation. Therefore, they had a short follow-up period ( $<3$ months) and a lack of pre-dialysis care by a nephrologist, so they had a high tendency to refuse dialysis. Third, even though the surrogate outcome of patient knowledge of and confidence in PD increased after video counseling, there was no significant difference between the customized and conventional video counseling groups. The decision to undergo dialysis depends on many abstract aspects, such as knowledge of and confidence in dialysis; personal values and beliefs; and feelings toward life, suffering, death, and other patient experiences [6,12-14]. In-depth interviews on this issue will be needed to further evaluate the true situation.

Our study aimed to address the hypothesis that educational content to aid in PD decision-making customized to patient context could help patients accept PD in a timely manner. The evidence from the dialysis decisionmaking aids found that an education booklet with a highquality design based on science could more efficaciously help patients understand the treatment options that fit their lifestyles [15]. It is critical to determine the essential content in dialysis decision-making aids that allow patients and their caregivers to make better dialysis decisions in a timely manner; however, this essential content is still unknown. To the best of our knowledge, our study was the first randomized controlled trial to investigate this question in the PD-first policy setting. Another advantage of our study was that there were no lost participants.

However, our study did have several limitations. First, the nurse involved in the counseling process and who obtained the patients' final dialysis decisions was not blinded to the video counseling groups, so bias may have occurred in both the counseling process and outcome assessment. Second, we calculated the minimum sample size based on our optimism that the acceptance rate of PD catheter insertion with conventional catheter insertion would increase from $55 \%$ to $80 \%$ with $80 \%$ power. However, the acceptance rate in the study increased to approximately $70 \%$ in both groups, so this study was underpowered. Third, there were contamination factors for approximately two weeks during the end of PD decisionmaking before scheduling of PD catheter insertion. Some patients accepted PD after the counseling protocol but did not undergo PD catheter insertion on schedule. These patients may have influenced other people during these two weeks and thus may have served as contamination factors. Finally, due to their often late presentation and lack of counseling, patients who respond that they will accept PD may not always proceed with a plannedstart dialysis. Some patients require temporary hemodialysis to improve uremic symptoms and only then can undergo PD catheter insertion.

It is well-known that attending a pre-dialysis education program improves the likelihood that CKD patients will choose PD as a mode of dialysis and illustrates the 
importance of integrating an education program into the CKD clinic $[16,17]$. Our study added new evidence that customizing the content of these education programs to patient context may be beneficial to dialysis decisionmaking in the PD-first setting. Moreover, our study explicitly examined the views and perceptions of patients who were hesitant to start dialysis, even when CKD symptoms occurred.

We encourage each PD center to focus on the counseling and educational process for PD decision-making at an appropriate time. This will lead to a planned start of PD. In addition, video counseling that is customized to the local context should be implemented in each PD center, especially in the PD-first policy setting. Finally, the method of educational aid for dialysis decision-making in the PD-first policy is still a challenge and needs to be improved. A randomized controlled trial that compares only video counseling with other distinct methods (e.g., focus groups, patients sharing their experiences, motivational interviews, or interactive interviews regarding the rate of acceptance of PD or the rate of planned-start dialysis in stage $5 \mathrm{CKD}$ ) is urgently needed. Furthermore, in-depth interviews to determine the reasons for deferring PD are necessary for a feedback loop to improve the counseling process.

In conclusion, our study showed that customized video counseling in terms of patient context was not significantly different from conventional video counseling for PD decision-making along with PD catheter insertion in stage 5 CKD patients under the PD-first policy. Nevertheless, we encourage each PD center to focus on counseling and the educational process to improve the acceptance rate of PD among patients, which will then lead to a higher rate of planned-start PD. Evidence supporting counseling methods for PD and the appropriate educational content required for PD counseling are urgently needed to improve dialysis decision-making in stage 5 CKD patients.

\section{Conflicts of interest}

All authors have no conflicts of interest to declare.

\section{Acknowledgments}

We would like to thank the staff of the PD Unit at the
Department of Medicine, Maharat Nakhon Ratchasima Hospital for supporting this project.

\section{Authors' contributions}

Watanyu Parapiboon and Wannapat Pitsawong participated in the data collection and wrote the manuscript. Watanyu Parapiboon, Wannapat Pitsawong, and Lalana Raegasint participated in the study design and performed the statistical analysis. Watanyu Parapiboon participated in the conception, analysis, and interpretation of data. Laddaporn Wongluechai and Kanin Thammavaranucupt provided intellectual content of critical importance to the work and technical support. Wannapat Pitsawong and Lalana Raegasint participated in the study design and coordination and helped to draft the manuscript. All authors read and approved the final manuscript.

\section{References}

[1] Stevens PE, Levin A; Kidney Disease: Improving Global Outcomes Chronic Kidney Disease Guideline Development Work Group Members. Evaluation and management of chronic kidney disease: synopsis of the kidney disease: improving global outcomes 2012 clinical practice guideline. Ann Intern Med 2013;158:825-830.

[2] Lacson E Jr, Wang W, DeVries C, et al. Effects of a nationwide predialysis educational program on modality choice, vascular access, and patient outcomes. Am J Kidney Dis 2011;58:235-242.

[3] Shukla AM, Hinkamp C, Segal E, et al. What do the US advanced kidney disease patients want? Comprehensive pre-ESRD Patient Education (CPE) and choice of dialysis modality. PLoS One 2019;14:e0215091.

[4] Chan CT, Blankestijn PJ, Dember LM, et al.; Conference Participants. Dialysis initiation, modality choice, access, and prescription: conclusions from a Kidney Disease: Improving Global Outcomes (KDIGO) controversies conference. Kidney Int 2019;96:37-47.

[5] Narva AS, Norton JM, Boulware LE. Educating patients about CKD: the path to self-management and patientcentered care. Clin J Am Soc Nephrol 2016;11:694-703.

[6] Cassidy BP, Harwood L, Getchell LE, Smith M, Sibbald SL, Moist LM. Educational support around dialysis modality decision making in patients with chronic kidney disease: qualitative study. Can J Kidney Health Dis 2018;5: 
2054358118803323.

[7] Tennankore KK, Soroka SD, Kiberd BA. The impact of an "acute dialysis start" on the mortality attributed to the use of central venous catheters: a retrospective cohort study. BMC Nephrol 2012;13:72.

[8] Paek SC, Meemon N, Wan TT. Thailand's universal coverage scheme and its impact on health-seeking behavior. Springerplus 2016;5:1952.

[9] Tantivess S, Werayingyong P, Chuengsaman P, Teerawattananon Y. Universal coverage of renal dialysis in Thailand: promise, progress, and prospects. BMJ 2013;346:f462.

[10] Turner RC, Carlson L. Indexes of item-objective congruence for multidimensional items. Int J Test 2003;3:163-171.

[11] Whitley E, Ball J. Statistics review 4: sample size calculations. Crit Care 2002;6:335-341.

[12] Morton RL, Tong A, Howard K, Snelling P, Webster AC. The views of patients and carers in treatment decision making for chronic kidney disease: systematic review and thematic synthesis of qualitative studies. BMJ 2010;340:c112.
[13] Visser A, Dijkstra GJ, Kuiper D, et al. Accepting or declining dialysis: considerations taken into account by elderly patients with end-stage renal disease. J Nephrol 2009;22:794799.

[14] Lee A, Gudex C, Povlsen JV, Bonnevie B, Nielsen CP. Patients' views regarding choice of dialysis modality. Nephrol Dial Transplant 2008;23:3953-3959.

[15] Winterbottom AE, Gavaruzzi T, Mooney A, et al. Patient acceptability of the Yorkshire Dialysis Decision Aid (YoDDA) booklet: a prospective non-randomized comparison study across 6 predialysis services. Perit Dial Int 2016;36:374-381.

[16] Chan CT, Wallace E, Golper TA, et al. Exploring barriers and potential solutions in home dialysis: an NKF-KDOQI conference outcomes report. Am J Kidney Dis 2019;73:363371.

[17] Goovaerts T, Jadoul M, Goffin E. Influence of a pre-dialysis education programme (PDEP) on the mode of renal replacement therapy. Nephrol Dial Transplant 2005;20:18421847. 